\title{
Aggregating probabilistic predictions of the safety, efficacy, and timing of a COVID-19 vaccine
}

\author{
Thomas McAndrew, ${ }^{1, *}$ Juan Cambeiro, ${ }^{2}$ and Tamay Besiroglu ${ }^{2}$ \\ ${ }^{1}$ College of Health, Lehigh University, Bethlehem, Pennsylvania, United States of America \\ ${ }^{2}$ Metaculus
}

(Dated: June 3, 2021)

Safe, efficacious vaccines were developed to reduce the transmission of SARS-CoV-2 during the COVID-19 pandemic. But in the middle of 2020, vaccine effectiveness, safety, and the timeline for when a vaccine would be approved and distributed to the public was uncertain. To support public health decision making, we solicited trained forecasters and experts in vaccinology and infectious disease to provide monthly probabilistic predictions from July to September of 2020 of the efficacy, safety, timing, and delivery of a COVID-19 vaccine. We found, that despite sparse historical data, a consensus - a combination of human judgment probabilistic predictions - can quantify the uncertainty in clinical significance and timing of a potential vaccine. The consensus underestimated how fast a therapy would show a survival benefit and the high efficacy of approved COVID-19 vaccines. However, the consensus did make an accurate prediction for when a vaccine would be approved by the FDA. Compared to individual forecasters, the consensus was consistently above the $50^{\text {th }}$ percentile of the most accurate forecasts. A consensus is a fast and versatile method to build probabilistic predictions of a developing vaccine that is robust to poor individual predictions. Though experts and trained forecasters did underestimate the speed of development and the high efficacy of a SARS-CoV-2 vaccine, consensus predictions can improve situational awareness for public health officials and for the public make clearer the risks, rewards, and timing of a vaccine.

*mcandrew@lehigh.edu 


\section{INTRODUCTION}

SARS-CoV-2 - the infectious agent that causes COVID-19 - spread rapidly throughout the world and is responsible for millions of deaths [1-5]. The pandemic has negatively impacted societal, psychological, and economic factors, made those with comorbidities more susceptible to disease, and may have increased health inequities $[6-15]$.

In response, public health officials have offered guidance on non-pharmaceutical interventions (NPI), and have tracked and forecasted the transmission of SARS-CoV-2. However both tracking and NPI efforts have had mixed results [16-23]. Immunization has been shown to be an effective and long term solution to reducing the burden of many different diseases [24, 25]. Computational models predict widespread delivery of effective vaccines in addition to continued adherence to non-pharmaceutical interventions have the potential to reduce the impact of SARS-CoV-2 and COVID-19 [26-28].

Three vaccines to protect against COVID-19 disease have been authorized on an emergency basis by the Food and Drug Administration (FDA): a vaccine made by Pfizer and BioNTech with a reported efficacy of 95\%, a vaccine produced by Moderna with an efficacy of $94 \%$, and a single-dose vaccine produced by Johnson and Johnson with an efficacy of $66 \%$ [29-31]. Similar to tracking the transmission of disease, the U.S. Centers for Disease Control and Prevention (CDC) tracks the number of allocated, delivered, and administered COVID-19 vaccines in the U.S. to improve the situational awareness of public health decision-makers [32].

But in the middle of 2020 there was no approved COVID-19 vaccine. Vaccines were still in progress and whether a vaccine would be authorized, how safe and effective a vaccine would be, and how fast a vaccine could be produced and delivered to the public was uncertain.

Our goal was to aggregate probabilistic predictions of the safety, efficacy, and timelines of a COVID-19 vaccine from trained, generalist forecasters and experts in vaccinology and infectious disease into a single consensus prediction and produce reports to support public health decision-making. We used an online human judgment forecasting platform to collect probabilistic predictions from June 2020 to September $2020[33]$.

Computational ensembles during the COVID-19 pandemic have been used to guide the selection and continued evaluation of trial sites for vaccine efficacy studies [34] and have been used to predict the number of individuals fully vaccinated [35]. Human judgment has been applied to predict both epidemiological targets of COVID-19 [36] and aspects of the various vaccination efforts against COVID-19 [37, 38].

A multi-model ensemble combines predictive distributions from individual models to produce a single predictive distribution. Because an ensemble can combine models trained on different sets of data and with different underlying assumptions, an ensemble has the potential to produce a more accurate, better calibrated predictive distribution compared to the individual models that contributed to the ensemble [39, 40]

Aggregating predictions from subject-matter experts and generalist forecasters offers an alternative to computational modeling and can be useful when historical data is sparse, predictions are needed for a small number of targets, and when information needed by public health officials can change rapidly [36, 39, 41, 42]. Human judgment has been applied to generate probabilistic forecasts in several different domains [41-45]. In the field of infectious disease, human judgment has predicted rates of US influenza-like illness [46], West-Nile virus [47] and Malaria [48], and epidemiological targets of the early US trajectory of COVID-19 [36].

Forecasts of vaccine characteristics have the potential to impact public, public health official, and vaccine research and development decision making under uncertainty. Efficacy and safety of vaccine may determine whether an individual volunteers to be inoculated [49-52]. For public health officials, predictions of the 
time to approval and time to manufacture a vaccine serve as valuable input in supply chain management, including logistics planning, inventory management, material requirements planning [53-55]. There is also a need from those in research and development for probabilistic predictions to help determine which vaccine platforms and pathways should be pursued before others [56-59].

To the best of our knowledge, this work is the first to generate ensemble probabilistic predictions from expert and generalist forecasters on COVID-19 vaccine development and share these with the public and public health decision-makers from June 2020 through September 2020, before the first approved COVID-19 vaccine.

\section{METHODS}

\section{II.1. Survey timeline}

Subject matter experts (SMEs) and trained forecasters (definition below) participated in four surveys from June 15th, 2020 to August 30th, 2020. SMEs and forecasters were asked to predict aspects of safety, efficacy, and delivery of a COVID-19 vaccine (see [33] to view all four summary reports, questions asked of forecasters and collected prediction data used in this work).

Subject matter experts and trained forecasters were solicited by sending personal emails (see Supp. S1 for a template email sent to subject matter experts). Solicitation started on June 3, 2020 and ended on July 8, 2020 .

The first two weeks of each month (the 1st to the 14th) was used to develop questions that could address changing information about a COVID-19 vaccine.

Forecasters received a set of questions on the 15 th of each month and from the 15th to the 25th could submit predictions using the Metaculus platform [60]. Forecasters made a first prediction and, as many times as they wished, could revise their original prediction between the 15th and 25th. To reduce anchoring bias [61], between the 15th and the 20th forecasters made predictions without knowledge of other forecaster's predictive densities. From the 20th to the 25th a consensus predictive density - an equally weighted combination of predictive densities from subject matter experts and trained forecasters - was available to forecasters.

\section{II.2. Forecasters}

We defined a subject matter expert as someone with training in the fields of molecular and cellular biology, microbiology, virology, biochemistry, and infectious diseases, and who has several years of experience in vaccine, antiviral, and/or biological research related to infectious agents and kept up-to-date with vaccine and antiviral research specifically focused on SARS-CoV-2/ COVID-19. Subject matter experts were trained in biological sciences but were not required to have had prior experience making accurate, calibrated probabilistic predictions.

We defined a trained forecaster as someone who ranked in the top 1\% out all Metaculus forecasters (approximately 15,000 forecasters), according to a Metaculus point system, and who has made consistent predictions on the Metaculus forecasting platform for a minimum of one year. Past work has shown that individuals who make consistent and accurate forecasts of one set of targets may be able to apply their forecasting skills 
to other targets [62-64]. Trained forecasters were not required to have a background in vaccines, biology, or infectious diseases, but they were required to have a history of making accurate predictions across a variety of topics and forecasting tournaments.

\section{II.3. Vaccine-related questions}

Forecasters were asked questions that fell into four categories: safety, efficacy, timing and delivery, and urgent matters (see Suppl. Table S1 for a list of all questions asked over all four surveys).

All questions asked of forecasters contained background information related to the target of interest, the question itself, and a detailed paragraph that described how we will determine the true, final value used to score predictive distributions.

Safety questions asked about survival rates related to vaccination. In particular, forecasters were asked to predict the probability that more than 10 patients experience a serious adverse event from a COVID-19 vaccine within one year of the date that vaccine was approved, and when a randomized controlled trial that tests a COVID-19 vaccine compared to a suitable control will show a statistically significant survival benefit.

Efficacy questions aimed to estimate the relative difference in attack rate between those vaccinated and those unvaccinated for the (at the time) ongoing trial to test the ChAdOx1 vaccine, for an approved vaccine under standard regulatory guidance or an emergency use authorization, and for a vaccine using one of several different viral platforms.

Timing and delivery questions asked forecasters to predict when the first SARS-CoV-2 vaccine would be approved in the United States or in the European Union, and to compare differences between the date that the FDA would approve a SARS-CoV-2 vaccine under a standard regulatory process versus expedited (emergency) process. Forecasters were also asked to predict the elapsed time between when a vaccine would be approved and when 100M doses would be manufactured.

In addition to safety, efficacy, and timing and delivery, forecasters were asked to predict the number of vaccine candidates in both clinical and pre-clinical development.

Questions were designed to communicate the risks (efficacy and safety) of being inoculated with a COVID-19 vaccine candidate; provide public officials a potential timeline of vaccine approval so that they can prepare; present objective, falsifiable predictions of vaccine development from an expert crowd; and decrease potential misinformation available to the public.

\section{II.4. Forecasting platform}

Forecasters submitted predictive densities by accessing the Metaculus platform (Metaculus), an online forecasting platform that allows users to submit predictive densities and comments related to a proposed question. Metaculus stores individual predictions and comments for each question and when the ground truth for a question is available the platform scores individual predictions and keeps a history of each forecaster's average score across all questions for which they have submitted predictions. The Metaculus platform allows participants to visualize their proposed predictive density both as probability density and cumulative density functions.

A private subdomain was created on Metaculus that allowed only subject matter experts and select trained 
forecasters to submit predictions and comments on these COVID-19 vaccine questions. Forecasters were encouraged, but not required, to answer all questions. When a user accesses Metaculus they are presented with a list of questions for which they can submit probabilistic predictions. For each question, the forecaster was presented background information about the specific question, including resources judged by the authors to be relevant and informative. Each question also contained a detailed statement of the resolution criteria - the criteria that describes, as precisely as possible, how the ground-truth would be determined.

Below the question, forecasters are presented with a tool to form a predictive density as a weighted mixture of up to five logistic distributions (See Fig. 1). The logistic distribution resembles a normal distribution but has heavier tails.

\section{II.5. Individual predictions}

Experts and trained forecasters submit a probabilistic density as a convex combination of up to five logistic distributions. Specifically, the ensemble probabilistic prediction $f_{m}$ for the $m^{\text {th }}$ forecaster is given by:

$$
\begin{gathered}
f_{m}(x)=\sum_{j=1}^{5} \alpha_{j} p_{j}\left(x \mid \mu_{j}, \sigma_{j}\right) \\
\text { where } p_{j}\left(x \mid \mu_{j}, \sigma_{j}\right)=\frac{e^{-\left(x-\mu_{j}\right) / \sigma_{j}}}{\sigma_{j}\left(1+e^{-\left(x-\mu_{j}\right) / \sigma_{j}}\right)^{2}}, \alpha_{j} \geq 0, \sum_{j=1}^{5} \alpha_{j}=1
\end{gathered}
$$

When generating a prediction, participants specify at minimum one logistic distribution by moving a slider that corresponds to $\mu$ and compressing or expanding the same slider that corresponds to $\sigma$. If a participant decides to add a second (or $3^{\text {rd }}, 4^{\text {th }}$, and $5^{\text {th }}$ ) logistic distribution they can click "add a component" for a second slider allowing the participant to "shift and scale" this additional logistic distribution. Two "weight" sliders also appear under each "shift and scale" slider that allows the participant to control the weights $\left(\alpha_{1}, \alpha_{2}, \cdots, \alpha_{5}\right)$ of each individual logistic distribution.

Forecasters can assign probabilities over a domain of pre-specified possible outcomes. The domain $(D)$ is typically a closed interval of the real number line where the lower $(L)$ and upper bounds $(U)$ of the interval are chosen to contain all possible outcomes $(D=[L, U])$. In some cases an additional outcome allows forecasters to assign probability to an open-ended outcome falling outside the upper or lower bound of the domain $(D=\ell \cup[L, U] \cup v)$. For example, for the question "When will a COVID-19 vaccine show an efficacy of greater than or equal to 70\%?" a forecaster could assign a probability density from Aug, 2020 to April, 2023 and to the outcome "later than April, 2023".

A submitted individual forecaster ensemble probabilistic prediction is stored by the forecasting platform as an array of 200 density values evaluated at 200 equally spaced points from the minimum to maximum allowable values (i.e. 200 equally spaced values in $[L, U]$ ). For questions with no upper or lower bound, densities are still stored over the interval $[L, U]$ with the understanding that because the interval $[L, U]$ does not cover all possible events the probability over $[L, U]$ is less than one. 


\section{II.6. Consensus building}

A consensus predictive density is a convex combination (often called a linear pool [65]) of probabilistic predictions from individuals

$$
f(x)=\sum_{m=1}^{M} \pi_{m} f_{m}(x)
$$

where $M$ is the number of forecasters who contributed a predictive distribution and $f_{m}$ is the $m^{\text {th }}$ forecaster's predictive density with an associated consensus weight $\pi_{m}$. The sum of weights for all $M$ forecasters must sum to one $\left(\sum_{m=1}^{M} \pi_{m}=1\right)$. For all consensus forecasts we chose to assign equal weights to each forecaster (i.e. $\left.\pi_{m}=\frac{1}{M}\right)$, as we had little a-priori reason to assign differential weights to participants.

\section{II.7. Scoring}

We chose to score forecasts using the logarithmic $(\log )$ score $[66,67]$. The log score assigns the logarithm of the density value corresponding to the eventual true value $(t)$ of a target of interest.

$$
\log \text { score }=\log [f(t)]
$$

where $f$ is the predictive density submitted by an individual forecaster or consensus. Log scores take values from negative to positive infinity. The worst possible log score a forecaster can receive is negative infinity (earned when the density assigned to the actual outcome value is zero), and the best possible score is positive infinity (earned when the density assigned to the actual outcome approaches positive infinity).

The $\log$ score is a proper scoring rule. A proper scoring rule is optimized when a forecaster submits the true density over potential values of a target, disincentivizing a forecaster from submitting a predictive density that does not accurately represent their true uncertainty over potential outcomes [68,69].

Scaled ranks were also reported for logscores. Given a set of $N \log$ scores, the scaled rank assigns a value of $1 / N$ to the smallest $\log$ score, a value of $2 / N$ to the second smallest log score, and so on, assigning a value of 1 to the highest log score.

\section{II.8. Statistical inference and testing}

A mixed effects regression model was fit to log scores from experts, trained forecasters, and consensus models (considered a forecasters) for all questions with ground truth data. The model is

$$
\begin{aligned}
L_{i, q} & \sim \mathcal{N}\left(\beta_{0}+\beta_{q}+\beta_{\text {expert }} E+\beta_{\text {consensus }} C, \sigma^{2}\right) \\
\beta_{q} & \sim \mathcal{N}\left(0, \sigma_{q}^{2}\right)
\end{aligned}
$$

where $L_{i q}$ is the $\log$ score generated by forecaster $i$ who made a prediction for question $q, E$ is a binary variable that identifies whether a forecaster was an expert $(E=1)$ or not $(E=0), C$ is a binary variable 
that indicates whether a forecaster was a consensus model $(C=1)$ or not $(C=0)$. and $\beta_{q}$ is a normally distributed, random intercept, with standard deviation $\sigma_{q}$ that accounts for the tendency for $\log$ scores to be clustered within each question.

All statistical hypothesis tests are two-sided and a pvalue less than 0.05 is considered statistically significant.

\section{II.9. Comments and information exchange}

Before, during, and after a question is available for forecasting, participants can post comments. Comments are used to clarify question text, for a forecaster to explain their rationale behind a forecast, and for signaling other potential forecasters about a potentially important data source (forecasters can notify one another by adding an "@" to a specific forecaster's username).

Forecasters can also interact with one another through a consensus distribution generated by Metaculus which was revealed for each question on the 25 th of each month, approximately half way through the total time left to make predictions. The consensus distribution was updated after the 25 th as individuals made new predictions or if an individual revised their previous prediction.

\section{RESULTS}

\section{III.1. Participation and response rate of the crowd}

Four monthly surveys were conducted in June, July, August, and September of 2020. A total of 10 individual experts and 11 trained forecasters participated in at least one of the four surveys. On average 6 experts participated (made at least one prediction for one question) per survey and an average of 8 trained forecasters participated per survey. The median number of hours after the survey was open until the first prediction by an expert was 12 hours and 9 hours for trained forecasters, and the last prediction by an expert was on average 6 hours and for a trained forecaster 2 hours before survey close.

Experts and trained forecasters (the crowd) were asked on average 6.5 questions and made on average 132.5 unique and revised predictions per survey. In June we asked 6 questions and received 154 total (unique and revised) predictions (26 predictions per question), in July we asked 7 questions and received 148 predictions (21 predictions per question), in August we asked 8 questions and received 153 predictions (19 predictions per question), and in September we asked 5 questions and received 75 predictions (15 predictions per question).

Comments were made on 21 out of 26 questions (80.7\%) with an average of 2.2 comments per question across all four surveys and a maximum number of comments of 8 on the question asked in June "When will a SARS-CoV-2 vaccine candidate demonstrate $\geq 70 \%$ efficacy?".

\section{III.2. Efficacy}

The consensus median prediction for when a vaccine would show an efficacy above $70 \%$ was Aug. 13, 2021 (IQR: [Mar. 2021, Feb., 2022]) and the consensus assigned a 0.12 probability to a COVID-19 vaccine 
reporting an efficacy of $70 \%$ or higher between June 30th, 2020 and Dec. 10, 2020. The consensus median prediction for the efficacy of the ChAdOx1 vaccine was $55.2 \%$ (80CI: $[7.5 \%, 83.6 \%]$ ), the consensus expected a higher reported efficacy for a vaccine that underwent a standard regulatory process (median: $67 \%$; IQR: $[58 \%, 75 \%]$ ) compared to an expedited emergency use authorization (median: $50 \%$; IQR: [37\%, 63\%]), and the consensus assigned the highest median efficacy to a vaccine using a protein subunit platform (median: 69\%; IQR: $[54 \%, 79 \%])$ compared to a non-replicating viral platform, inactiviated virus platform, and DNA/RNA platform. We asked trained forecasters and experts eight questions, two in June, four in July, and two in August, related to the efficacy of a COVID-19 vaccine (Fig. 2) and received 160 predictions (20 predictions per question on average).

The consensus median prediction made in June, 2020 for when a vaccine would show an efficacy above $70 \%$ was Aug. 13, 2021, the mode was July 1, 2021, and the $90 \%$ confidence interval (CI) was [Oct., 2020, May, 2023] (Fig. 2A.). The consensus assigned a 0.85 probability to a COVID-19 vaccine with greater than or equal to $70 \%$ efficacy occurring after 2020 . The first vaccine to show an efficacy greater than or equal to $70 \%$ was the Pfizer-BioNTech vaccine, reporting an efficacy of $95 \%$ on Dec. 10, 2020.

The consensus median prediction made in June, 2020 for the efficacy of the ChAdOx 1 vaccine is $55 \%$ with a probability of 0.27 assigned to values between $60 \%$ and $80 \%$ and a probability of 0.13 assigned to an efficacy below $10 \%$ (Fig. 2B.). The reported efficacy of the ChAdOx1 vaccine was $61.2 \%$ [70].

The consensus mode prediction of efficacy made in July, 2020 was $60 \%$ for a vaccine produced using a nonreplicating viral platform, $65 \%$ for a vaccine produced using a DNA/RNA platform, $75 \%$ for a inactivated, and $77 \%$ for a protein sub-unit platform (Fig. 2C.). The probability assigned to an efficacy below $50 \%$ was 0.35 for a non-rep platform, 0.30 for a DNA/RNA platform, 0.25 for an inactivated platform, 0.19 for a protein sub-unit platform.

The consensus mode (median) prediction made in August, 2020 of the efficacy of a vaccine approved under a standard regulatory process was $66 \%(66 \%)$ and was $52 \%(50 \%)$ for a vaccine approved under an emergency use authorization (such as Operation Warp Speed) (Fig. 2D.). An efficacy of less than or equal to $50 \%$ was assigned by the consensus a probability of 0.16 for a vaccine approved under a standard regulatory process and a probability of 0.51 for a vaccine approved under an emergency use authorization.

\section{III.3. Safety}

The consensus median prediction, made on June 30th, for when a COVID therapy will show a significant difference in survival was March, 2021 (80CI: [Sept., 2020, Dec., 2022]), on July 27th the consensus mode prediction for when a significant survival effect would be reported for a therapy using monoclonal antibodies was Dec., 2020 (80CI: [Nov., 2020, March, 2023]) compared to a mode of Dec., 2020 (80CI: [Oct., 2020, June, 2022]) for an antiviral platform, and June, 2024 (80CI: [Feb., 2021, March, 2024]) for an orally administered treatment. The consensus assigned a probability of 0.67 to a greater than $80 \%$ chance of 10 or more serious adverse events caused by a SARS-CoV-2 vaccine (under a standard or emergency regulatory process). We asked trained forecasters and experts six questions, one in June, two in July, one in August, and two in September related to the safety of a COVID-19 vaccine (Fig. 3) and received 131 predictions (21 predictions per question on average)

The mode prediction made in June, 2020 of when a COVID-19 therapy would show a significant survival benefit was Oct., 2020 (Fig. 3A.). A probability of 0.35 was assigned to a vaccine showing a survival benefit from June 15th, 2020 to Dec. 31, 2020, a probability of 0.42 was assigned between the dates Jan. 1, 2021 and 
Dec. 31, 2021, and a probability of 0.23 after 2021 . July 17 th, 2020 was the $6^{\text {th }}$ percentile of the consensus predictive distribution. Dexamethasone - an anti-inflammatory treatment - was shown to have a significant benefit to survival on July 17th 2020 [71].

The consensus predictive density for when a monoclonal antibody platform would show a significant survival benefit had the smallest interquartile range (IQR: [Dec. 29, 2020, Oct. 15, 2021] ), second smallest IQR was for an antiviral platform (IQR: [Dec. 14, 2020, Sept. 16, 2021]), and largest IQR was for an orally administered therapy (IQR: [July, 2021, Jan., 2023]) (Fig 3B.). For the monoclonal antibody and antiviral platform, a 0.28 and 0.30 probability (respectively) was assigned to a survival benefit reported before Jan., 2021. The median prediction for when a monoclonal antibody platform would show a significant survival benefit was April, 2021, for an antiviral platform was April, 2021, and for an orally administered therapy was Jan., 2022.

The consensus median prediction, made on Sept. 30th, 2020, of the probability that greater than or equal to 10 serious adverse events are caused by a vaccine was 0.97 for a vaccine approved under standard regulatory conditions and 0.94 for a vaccine approved under an emergency use authorization.

\section{III.4. Time to approval}

A consensus of experts and trained forecasters made a median prediction in July, 2020 that a SARS-CoV-2 vaccine will be approved in the US or European Union in April, 2021 (80CI: [Oct., 2020, July, 2022]). In August the consensus median prediction for when a vaccine will be approved in the US under an emergency use authorization was Feb., 2021 (80CI: [Sept., 2020, Sept., 2022]) and in September the median prediction was Jan., 2021 (80CI: [Oct., 2020, Nov., 2021]) (Fig. 4). We asked trained forecasters and experts six questions, one in June, one in July, two in August, and two in September related to the timing of approval of a COVID-19 vaccine (Fig. 4) and received 120 predictions (20 predictions per question on average)

The consensus median prediction made on June 30th, 2020 for when a SARS-CoV-2 vaccine will be approved - either under a standard regulatory process or an emergency use authorization - in either the US or the European Union was May, 2021 (80CI: [Nov., 2020, Aug., 2022]) and the median prediction made on July 31st, 2020 was April, 2021 (80CI: [Oct., 2020, July, 2022]) (Fig. 4A.). There was a small difference in the median prediction between the survey issued in June and the survey issued in July (Diff. = 25 days), and a small difference in the number of days between the lower and upper bound of the $80 \%$ confidence interval made in June (637 days) and in July (639 days).

The consensus median prediction made in August, 2020 of when a SARS-CoV-2 vaccine would be approved in the US and under an emergency use authorization was Feb., 2021 (80CI: [Sept., 2020, Sept., 2022]) and for the consensus prediction made in September, 2020 was Jan., 2021 (80CI: [Oct., 2020, Nov., 2021]) (Fig. 4B. red). Under a standard regulatory process the consensus median prediction made in August, 2020 of when a SARS-CoV-2 vaccine would be approved was July, 2021 (80CI: [Dec.,2020, Oct., 2022]) and the consensus prediction made in September, 2020 was Oct., 2021 (80CI: [Jan., 2021, March, 2023]) (Fig. 4B. blue)

The difference in the days between the median prediction of when a vaccine candidate would be approved under an emergency use authorization vs a normal regulatory process was 148 days in August and 285 days in September. The September median prediction compared to the August median prediction of when a vaccine would be approved under an emergency use authorization was 26 days sooner (from Feb, 2020 to Jan 2020 ) and the September median prediction of when a vaccine would be approved under a normal regulatory process was 109 days later (from July to October, 2021). 


\section{III.5. Rate of production and delivery}

The consensus median prediction made in July, 2020 of when an approved vaccine in the US or EU would be administered to more than 100,000 people was Sept. 2021 (80CI: [Jan., 2021, Oct., 2022]). In Aug, 2020 the median prediction for the number of weeks after approval to produce $100 \mathrm{M}$ doses of a vaccine that uses a DNA/RNA platform was 18 (80CI: [5, 51]) and using a viral vector platform was 34 (80CI: [10, 72]) (Fig. 5). We asked trained forecasters and experts three questions, one in June and two in August related to speed to produce and administer a vaccine after (Fig. 5), and received 53 predictions (17 predictions per question on average)

The consensus assigned a 0.10 probability to $100 \mathrm{~K}$ doses of a vaccine administered by the end of 2020 and a 0.66 probability to $100 \mathrm{~K}$ doses administered by the end of 2021 (Fig. 5A.).

The consensus assigned a 0.27 probability to 10 weeks to manufacture $100 \mathrm{M}$ doses of a vaccine using a DNA/RNA platform compared to a 0.10 probability assigned to 10 weeks to produce $100 \mathrm{M}$ doses of a vaccine using a viral platform (Fig. 5B.). The difference in the median consensus prediction for the number of weeks to produce 100M doses for a vaccine using a DNA/RNA platform compared to a viral vector platform was 16 weeks.

\section{III.6. Predictive accuracy of individuals and the consensus}

The accuracy of a consensus of trained forecasters plus experts was in between the accuracy of a consensus generated from trained forecasters and the accuracy of a consensus generated from experts except for a single question where individual experts' accuracy was either below the 25th percentile or above the 75 th percentile (Fig. 6). Across all questions where the truth could be determined, the scaled ranks for a consensus of trained forecasters plus experts had a smaller interquartile range when compared to individual forecasters (Fig. 7).

The mean scaled rank for individual trained forecasters was 0.56 (80CI: $[0.18,0.94]$ ) and for individual subject matter experts was 0.48 (80CI: [0.08, 0.98]). The standard deviation of the scaled rank was 0.29 for trained forecasters and 0.34 for experts.

The mean scaled rank for the consensus generated from both trained forecasters and experts was 0.58 (80CI: [0.49, 0.63]) compared to a mean scaled rank of a trained forecasters only consensus of 0.56 (80CI: [0.43, $0.72])$, and for an experts only consensus of 0.53 (80CI: $[0.35,0.73])$. The consensus of trained forecasters and experts was more accurate than both the consensus of trained forecasters and experts for $1 / 6$ questions (proportion: 0.16, 80CI: [0,0.33]). Compared to a consensus of trained forecasters, a consensus of experts was more accurate for $3 / 6$ questions (proportion: $0.50,80 \mathrm{CI}$ : [0.16, 0.83]). A trained forecaster consensus was more accurate than a consensus of experts for $2 / 2$ efficacy questions (Fig. 6A.) and was more accurate when asked to predict the number of SARS-CoV-2 vaccine candidates that will be in human trials by Aug 1st, 2020 (Fig. 6D.). An expert consensus was more accurate than a consensus of trained forecasters when asked for the date when a COVID-19 therapy will show a survival benefit (Fig. 6B.) and for 2/2 questions related to the timing of approval of a SARS-CoV-2 vaccine.

The $25^{\text {th }}$ and $75^{\text {th }}$ percentiles for scaled rank was, from the widest interval to the smallest interval, [0.23, 0.84] for individuals, [0.48, 0.67] for a consensus of trained forecasters, [0.37, 0.67] for a consensus of experts, and $[0.53,0.60]$ for a consensus of trained forecasters plus experts (Fig. 7A.). The $25^{\text {th }}$ and $75^{\text {th }}$ percentiles for $\log$ scores, from the largest interval to smallest interval, was [0.42, 2.98] for all individuals, [0.65, 3.07] 
for a consensus of experts, $[0.98,2.96]$ for a consensus of trained forecasters plus experts, and $[1.24,2.90]$ for a consensus of trained forecasters (Fig. 7B.).

A consensus of trained forecasters plus subject matter experts scored above the $50^{\text {th }}$ percentile for $5 / 6$ questions while a trained forecaster consensus and an expert only consensus scored above the $50^{\text {th }}$ percentile on $3 / 6$ questions with ground truth.

Trained forecasters had the highest logscores on average, followed by consensus models, and then subject matter experts (Table I). Ninety five percent confidence intervals around the difference in logscores between subject matter experts and trained forecasters, and between consensus models and trained forecasters were large. We do not have enough data on forecast accuracy to conclude statistical significance at a type I error of $5 \%$ that trained forecasters made more accurate predictions than subject matter experts or consensus models.

\section{DISCUSSION}

A consensus of trained forecasters and experts in infectious disease and vaccinology provided predictions (i) that quantified uncertainty about the efficacy, safety, and timing of approval for a COVID-19 vaccine from July to September 2020, a time when the vaccine landscape and political atmosphere was rapidly changing; (ii) that were made when data on previous pandemics was sparse, likely relying instead on a combination of objective and subjective information, aspects of past pandemics that may have resembled the COVID-19 pandemic, and intuition; and (iii) that were fast and to the best of our knowledge before other human judgment or computational efforts to predict COVID-19 vaccine characteristics.

Expert and trained forecaster probabilistic predictions underestimated the speed of approval and high efficacy of a SARS-CoV-2 vaccine, and the consensus prediction of the date a COVID-19 treatment would show a survival benefit was much later compared to the truth.

The consensus assigned high probabilities to efficacy values above $70 \%$ for the Oxford/AstraZeneca vaccine and to vaccines based on four different vaccine technology platforms [72]. That said, the consensus median prediction made in Aug. 2020 of the efficacy of a vaccine approved under emergency authorization was 50\%, greatly underestimating the $95 \%$ efficacy of the Pfizer/BioNTech vaccine [29]. In addition to underestimating the efficacy of the first approved vaccine, consensus predictions for all questions related to efficacy assigned positive probabilities to values below $50 \%$, the threshold for approval stated by the FDA.

Consensus forecasts underestimated the speed of vaccine development. The Pfizer/BioNTech vaccine was approved Dec. 10th and on July 17th 2020 Dexamethasone - an anti-inflammatory treatment - was shown to have a significant benefit to survival [71]. The consensus in July, 2020 assigned a small probability to a vaccine approved before or on Dec. 10th, 2020, and the consensus median prediction made in June, 2020 for when a COVID-19 therapy would show a survival benefit was approximately eight months later than the true date. Late predictions of approval and when a vaccine would show a survival benefit are closer to the timeline of a traditional vaccine and could be because sparse objective data and subjective data caused forecasters to rely on past documentation related to vaccines that went through a standard regulatory pathway.

Often overlooked advantages to human judgment forecasting, compared to computational models, are the ability of a forecaster to transform textual data from the environment into a prediction and output textual data to backup their predictions while also directing other forecasters to potentially important information. For example, forecasters suggested political considerations may influence the timing of the vaccine authorization/approval process in the U.S. with one forecaster pointing out on June 16th that "pressure on the 
FDA to approve something will be enormous, and will start building once there are even preliminary phase 3 results." After our August survey closed and before the September survey opened, the President announced a vaccine might be approved before the 2020 election. We feel political events like this could have influenced forecasters because although the median prediction for when a SARS-CoV-2 vaccine will be approved in the US under emergency authorization shifted from Feb, 2021 to Jan, 2021 (One month sooner) in the midst of broadcasted political pressure about approving a vaccine, the median prediction for a vaccine to be approved under a standard regulatory process moved from July, 2021 to Oct., 2021 (3 months later). Humans are better positioned than computational models to synthesize textual data into their forecasts and, unlike computational models, can generate commentary that may present new information to others who plan to generate a forecast. Though the ability of forecasters to process textual information is an advantage in many cases, it can also mislead forecasts.

Individual predictive accuracy was higher for trained forecasters than for subject matter experts, and we found that aggregating individual predictions into a consensus can guard against inaccurate predictions made by individuals. Forecaster's individual accuracy was inconsistent and ranged from the bottom 25th percentile to the top 75th percentile across question type (efficacy, safety, timing) and from survey to survey. Compared to subject matter experts, trained forecasters had on average higher logscores. This may suggest predictive accuracy may depend more on forming good forecasting habits than on the subject matter.

Though a consensus was never the most accurate prediction, a consensus of trained forecasters and experts was in the top 50th percentile for five out of six questions. A consensus may not be the most accurate forecast, but a consensus can potentially guard against individual forecasters with poor accuracy.

In this work we are limited by a small number of questions we could ask forecasters, the number of questions that could be compared to the truth, and difficulties associated with human judgment.

A computational model, if possible to build, would be likely be able to make predictions for a large number of similar questions. However a forecaster often spends a significant amount of time to generate a forecast.

Only 23 percent (6/26) of the questions resolved, having true values we can use to to assess forecaster accuracy. A subset of questions will resolve over time but many questions will never resolve because the criteria to determine the truth (to resolve the question) may have been too strict. Many questions asked forecasters to predict the efficacy, safety, and timing of a vaccine that is granted a biologics license application (BLA), however, to date no SARS-CoV-2 vaccine has been granted a BLA.

The number of questions that are unable to resolve highlights that those who pose the questions are as important as those who submits predictions. Before forecasters begin to submit predictions, the author, when developing the question, must first predict whether or not they expect to obtain ground truth. For instance, there was uncertainty about whether or not the standard authorization process, emergency authorization process, or both would be used to approve vaccines. We expected vaccines to be approved by both pathways, but because only the emergency authorization process has been used any question limited to the standard regulatory process will never have a ground truth.

Forecasters were able to submit comments along with quantitative predictions, but a small percentage $(12 \%, 63$ comments divided by 530 predictions) of forecasters accompanied their predictions with text that explained the data they relied on and the rationale/model used to generate a probabilistic prediction. An ongoing challenge in human judgment forecasting is to solicit a prediction, data used to make the prediction, and a rationale.

Forecasts were made by trained forecasters and subject matter experts in a rapidly changing, chaotic environment and so, in line with representative design, we suggest our results can only be generalized to human judgment forecasting in a noisy environment without a large volume of historical data related to the questions 
asked of forecasters [73].

The purpose of this work was to develop predictive guidance for the public and public health officials and not to rigorously evaluate forecaster accuracy. Further experimental work is needed comparing subject matter experts to trained forecasters while varying task predictability and the number of linear and non-linear cues available in the environment to the forecaster. This work weighted equally predictive distributions from subject matter experts and trained forecasters, and further work is needed that explores differential weighting algorithms that may produce better accuracy.

Human judgement forecasting of progress towards a vaccine can be viewed as a tool to support primary preventative measures against an infectious agent. Probabilistic forecasts can target multiple audiences to support the public when making complex decisions about their health under uncertainty and support both short term and long-term health promotion practices implemented by public health officials. Because vaccination is a low frequency, voluntary event, forecasts did not aim to modify the public's sense of control or desire to engage in inoculation. Instead, forecasts made easily accessible online aimed to help ready individuals at all risk levels for vaccination and understand the impact of their decisions on their own and on other's health. Predictions from a consensus of the efficacy, safety, and timing of a vaccine may have been accurate enough to improve situational awareness for public health officials.

Consensus predictions based on human judgment are a promising tool to aid decision-making under the uncertainty of a rapidly evolving situation where there is little historical data, where data is only loosely applicable, and when timely risk communication is important. 


\section{TABLES}

\begin{tabular}{lccc}
\hline Variable & Coefficient & 95CI & pvalue \\
\hline Intercept (trained forecasters) & 2.10 & $(0.60,3.60)$ & 0.006 \\
Consensus models. & -0.01 & $(-0.51,0.50)$ & 0.983 \\
Subject matter experts & -0.14 & $(-0.56,0.29)$ & 0.531 \\
$\sigma_{\text {question }}$ & 3.42 & & \\
\hline
\end{tabular}

TABLE I: Table of coefficients, 95\% confidence intervals, and pvalues for a mixed effects model with log score as the dependent variable, three fixed categorical factors to identify log scores from trained forecasters, subject matter experts, or consensus models, and a random intercept by question. Trained forecasters have the highest average logscore followed by consensus models and subject matter experts. However, there is not enough evidence to conclude that these differences are statistically significant 
medRxiv preprint doi: https://doi.org/10.1101/2021.06.03.21258240; this version posted June 4, 2021. The copyright holder for this preprint (which was not certified by peer review) is the author/funder, who has granted medRxiv a license to display the preprint in perpetuity.

It is made available under a CC-BY-NC-ND 4.0 International license .

\section{FIGURES}

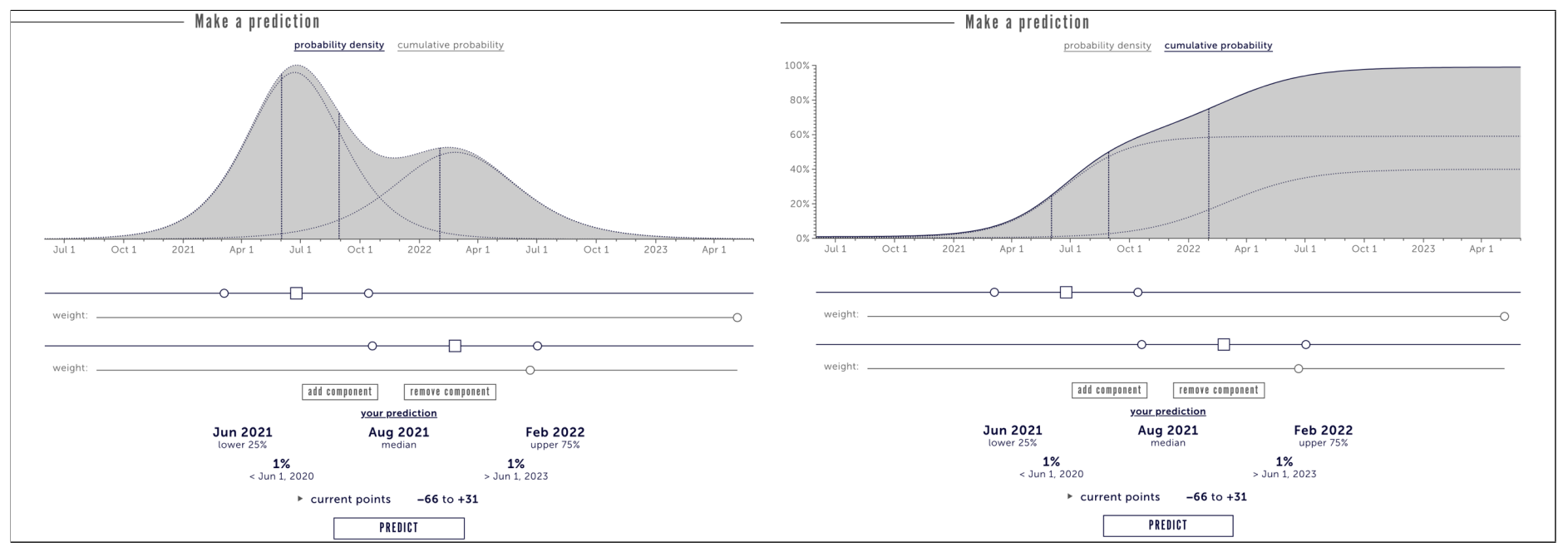

FIG. 1: The Metaculus prediction interface which records forecaster's probabilistic predictions. (Left) The probability density view (Right) The cumulative density view. The prediction interface allows users to shift and scale up to five logistic distributions (in this example two distributions are used) and increase or decrease the weight given to each distribution. The median, $25^{\text {th }}$, and $75^{\text {th }}$ percentiles are displayed to the forecaster. 
medRxiv preprint doi: https://doi.org/10.1101/2021.06.03.21258240; this version posted June 4, 2021. The copyright holder for this preprint (which was not certified by peer review) is the author/funder, who has granted medRxiv a license to display the preprint in perpetuity.

It is made available under a CC-BY-NC-ND 4.0 International license .
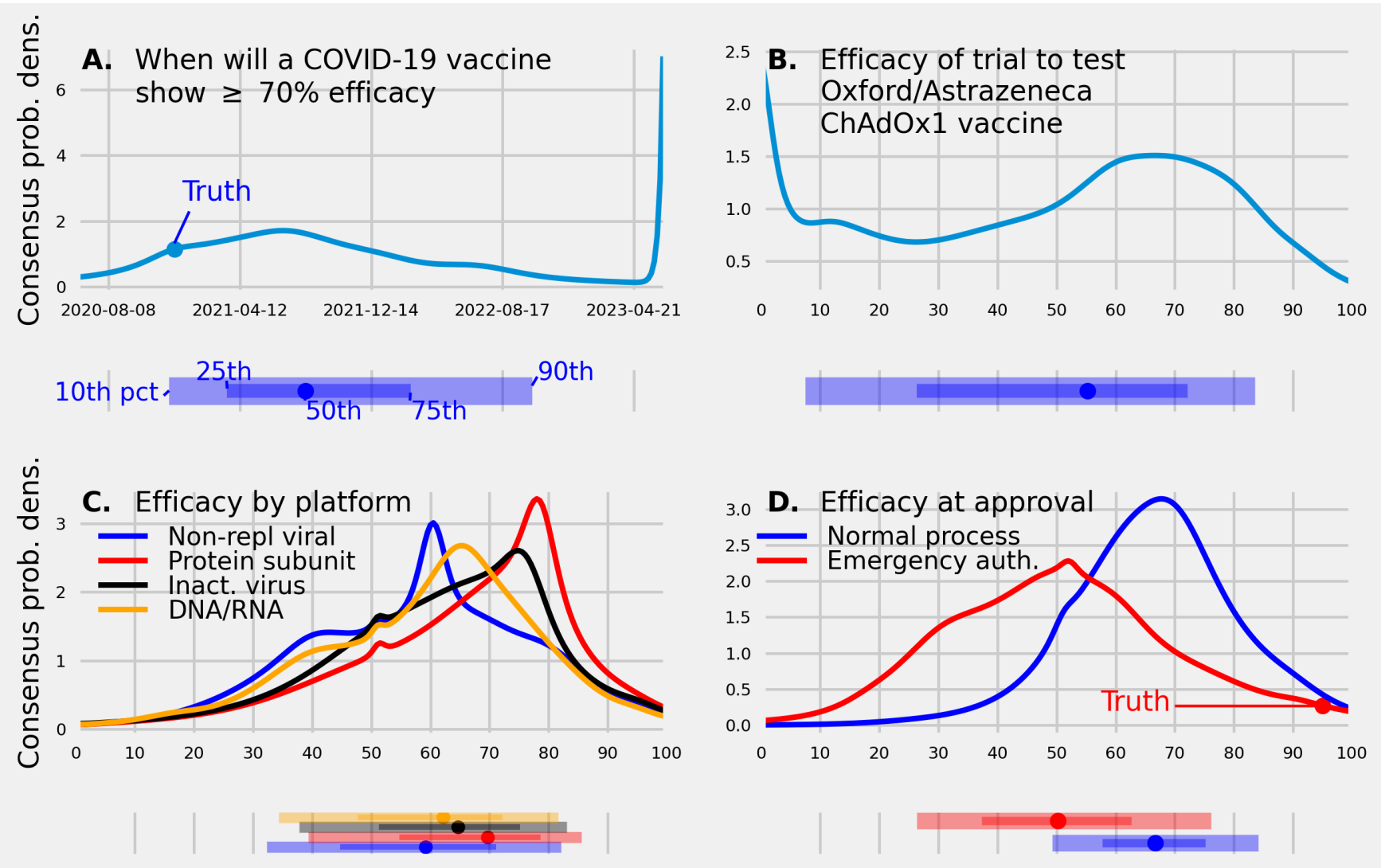

FIG. 2: (A.) A consensus predictive density made in June, 2020 of the date when a COVID-19 vaccine will demonstrate an efficacy of $70 \%$ or greater. The consensus assigned a 0.12 probability to a vaccine showing a $70 \%$ or greater efficacy by Dec. 10, 2020, the date the Pfizer and BioNTech vaccine was approved. (B.) A consensus predictive density made in June, 2020 of the efficacy reported from the trial testing the

ChAdOx1 vaccine (C.) A consensus predictive density made in July, 2020, of the efficacy of a vaccine based on four different platforms (D.) A consensus predictive density made in August, 2020 of the efficacy of a vaccine at approval under a standard regulatory process and emergency use authorization. Under each predictive density is the corresponding 10th, 25th, 50th (median), 75th, and 90th quantile. The true values, if available, are represented as a filled circle. A consensus of experts and trained forecasters made probabilistic predictions that compared vaccine efficacy between different regulatory mechanisms and between different vaccine delivery methods, gave a time-frame for when an efficacious vaccine will be approved, and made a testable prediction of the efficacy of a specific trial of interest. 
medRxiv preprint doi: https://doi.org/10.1101/2021.06.03.21258240; this version posted June 4, 2021. The copyright holder for this preprint (which was not certified by peer review) is the author/funder, who has granted medRxiv a license to display the preprint in perpetuity.

It is made available under a CC-BY-NC-ND 4.0 International license .
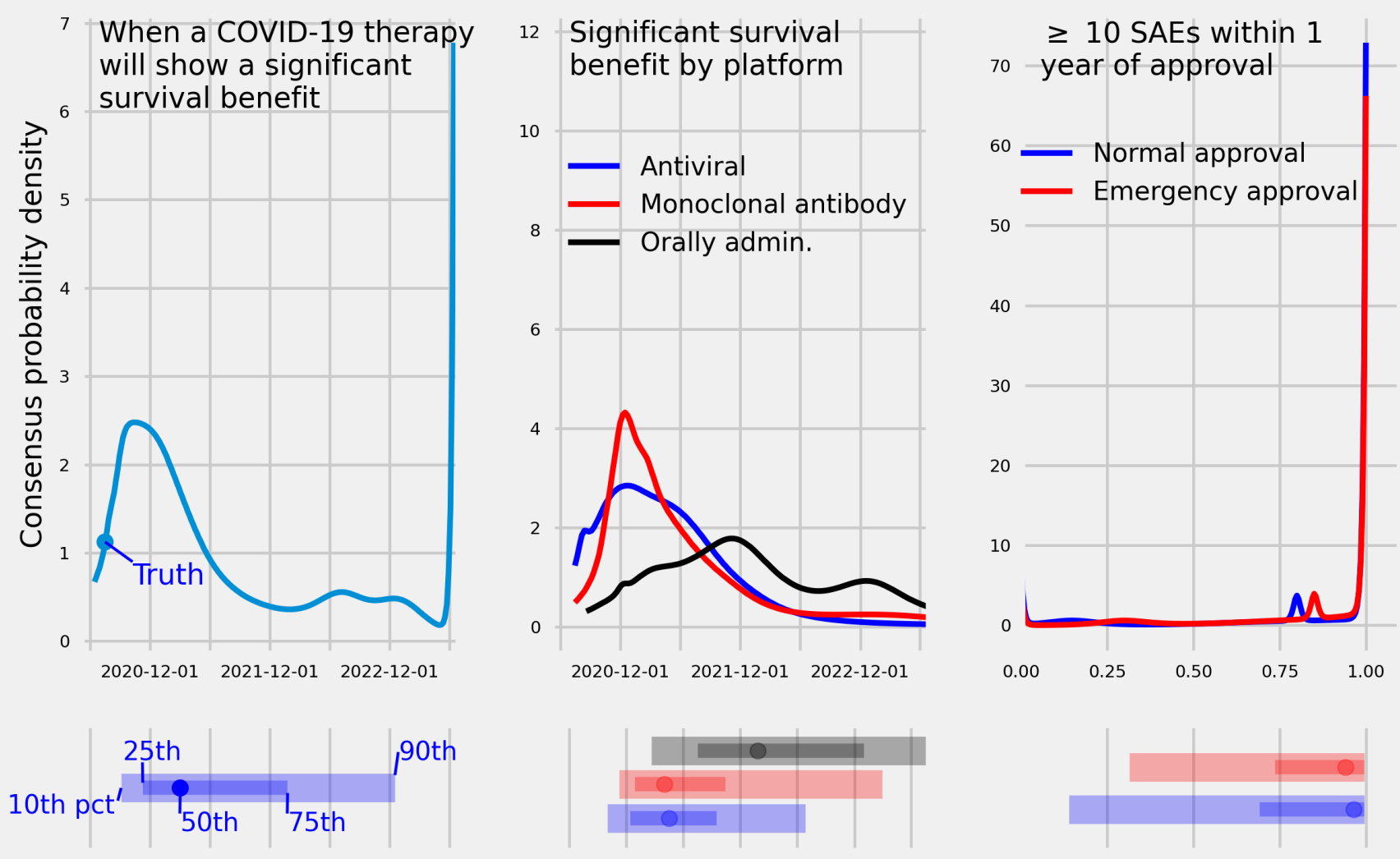

FIG. 3: (A.) A consensus predictive density made in June, 2020 over dates for when a COVID-19 therapy will show a significant survival benefit in a randomized clinical trial enrolling more than 200 patients.

(B.) Consensus predictive densities made in Aug., 2020 over dates when a SARS-CoV-2 vaccine will show a significant survival benefit in a randomized control trial enrolling more than 200 patients across three different viral platforms. (C.) Consensus predictive densities over the probability of ten or more serious adverse events within one year of the date of approval of the first SARS-CoV-2 vaccine approved through a standard regulatory process or emergency use authorization. Below each density is the $10^{\text {th }}, 25^{\text {th }}, 50^{\text {th }}$

(median), $75^{\text {th }}$, and $90^{\text {th }}$ percentile. True values, if available, are represented as a filled circle. The consensus was uncertain when a vaccine would show a survival benefit, assigning $80 \%$ confidence intervals that spanned close to two years for when a COVID-19 therapy would show a benefit. The consensus was certain at least 10 SAEs would be observed within one year of approval. 
medRxiv preprint doi: https://doi.org/10.1101/2021.06.03.21258240; this version posted June 4, 2021. The copyright holder for this preprint (which was not certified by peer review) is the author/funder, who has granted medRxiv a license to display the preprint in perpetuity.

It is made available under a CC-BY-NC-ND 4.0 International license .
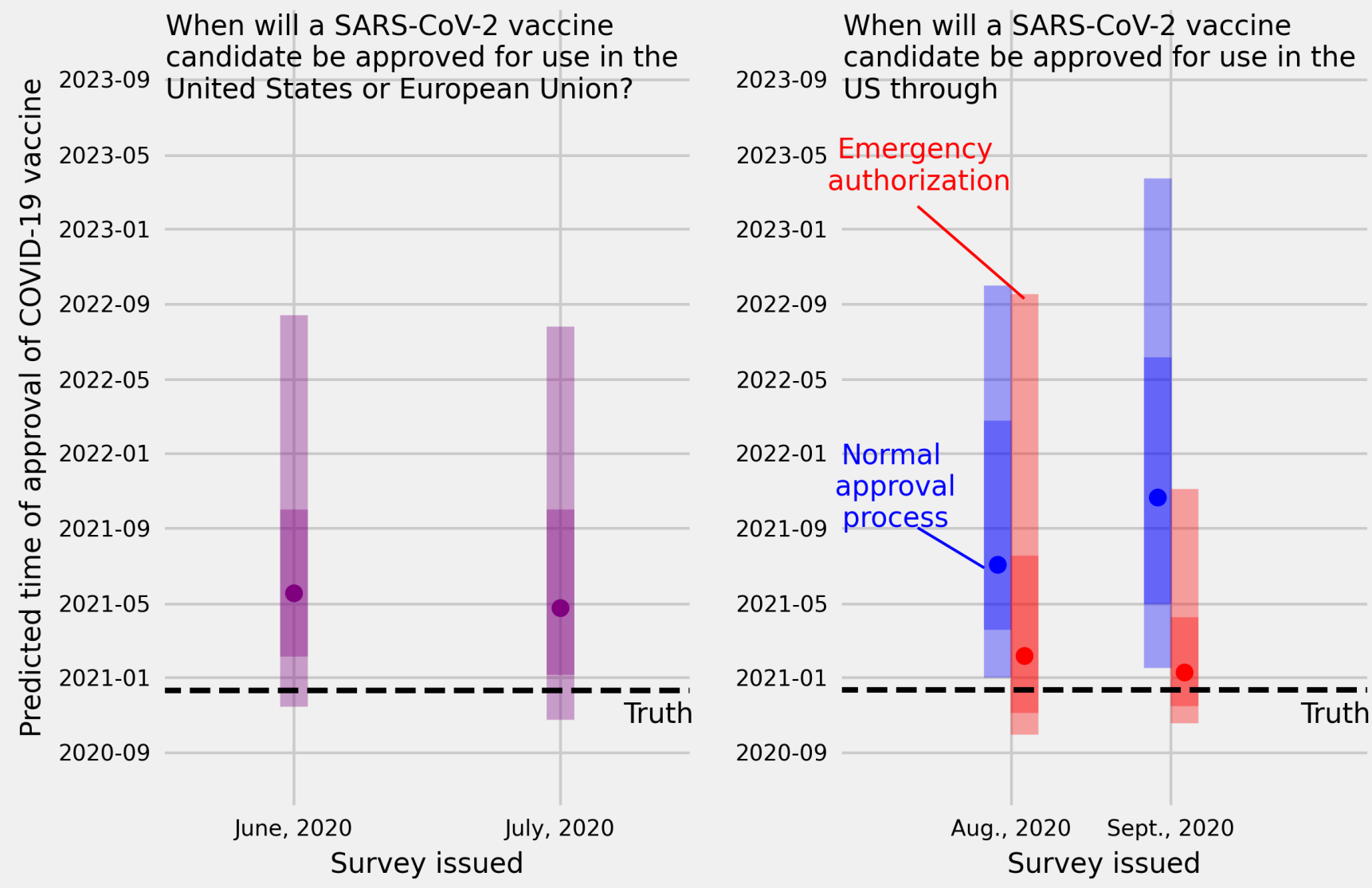

FIG. 4: (A.) Consensus predictive percentiles made in June and in July, 2020 for the date when SARS-CoV-2 vaccine will be approved for use in the US or European Union. (B.) Consensus predictive percentiles for the date a SARS-CoV-2 vaccine will be approved for use in the US through a standard regulatory process (blue) or an emergency use authorization (red). The consensus median predictions made

in June and July for when a SARS-CoV-2 candidate would be approved in the US or EU were many months later than the truth (May, 2020 and April, 2020 vs Dec., 2020). Consensus median predictions of the date of emergency approval of a SARS-CoV-2 vaccine in the US were Feb., 2020 (made in Aug., 2020) and Jan., 2020 (made in Sept., 2020) which were close to the true date Dec., 2020. An environmental cue, time between when the forecast was made and the truth, or how the question was asked, may have impacted predictive accuracy. 
medRxiv preprint doi: https://doi.org/10.1101/2021.06.03.21258240; this version posted June 4, 2021. The copyright holder for this preprint (which was not certified by peer review) is the author/funder, who has granted medRxiv a license to display the preprint in perpetuity.

It is made available under a CC-BY-NC-ND 4.0 International license .
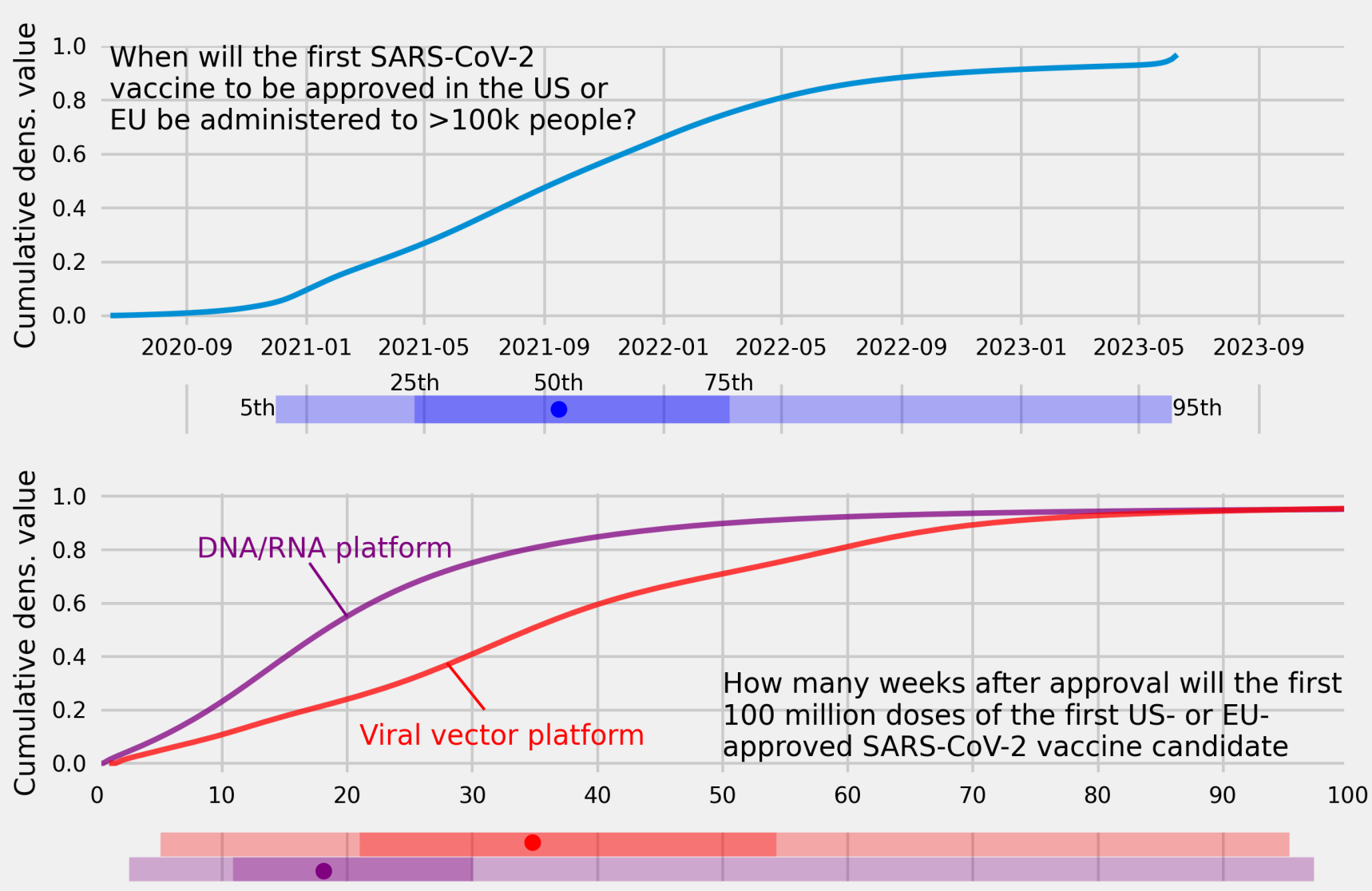

FIG. 5: (A.) A consensus cumulative predictive density of trained forecasters and experts over dates for when an approved SARS-CoV-2 vaccine in the US or European Union will be administered to more than 100,000 people. (B.) Consensus cumulative predictive densities of the number of weeks after approval needed to manufacture 100,000,000 doses of a vaccine using a DNA/RNA platform (purple dashed line) and a vaccine using a viral vector platform (red solid line). 


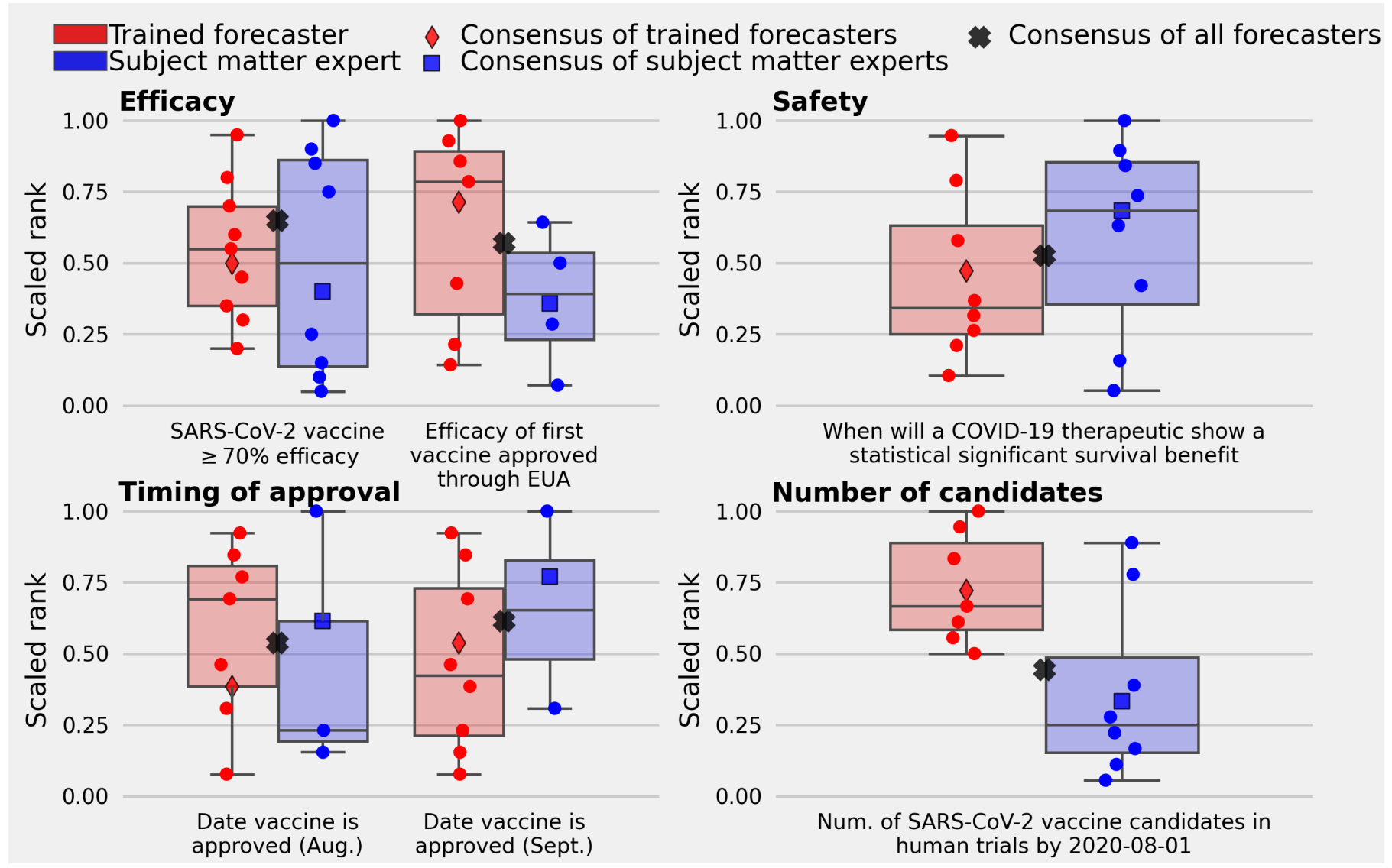

FIG. 6: The scaled rank for individual trained forecasters (red circles), subject matter experts (blue circles), and three consensus distributions: (i) a consensus of trained forecasters (red diamond), of subject matter experts (blue square), and the consensus of both trained forecasters and experts (black X) for 6 questions with ground truth. (A.) Two questions with ground truth relate to efficacy, (B.) one question is related to safety, (C.) two questions are related to the timing of vaccine approval, and (D.) one question asked forecasters for the number of vaccine candidates in human trials by Aug., 2020. The mean scaled rank of a consensus of trained forecasters and subject matter experts is 0.58 (80CI: [0.49, 0.63]), a consensus of trained forecasters only is 0.56 (80CI: [0.43, 0.72]), and a consensus of experts only is 0.53 (80CI: $[0.35,0.73])$. None of the three consensus predictions are the most accurate, however the trained forecaster plus expert consensus is in the top $50^{\text {th }}$ percentile for all but one question with ground truth. Aggregating trained forecasters and subject matter experts has the potential to guard against an individual forecast with poor accuracy. 
medRxiv preprint doi: https://doi.org/10.1101/2021.06.03.21258240; this version posted June 4, 2021. The copyright holder for this preprint (which was not certified by peer review) is the author/funder, who has granted medRxiv a license to display the preprint in perpetuity.

It is made available under a CC-BY-NC-ND 4.0 International license .

A.

Individuals

Consensus

of

trained forecasters

Consensus

of

experts

Consensus

of

all forecasters

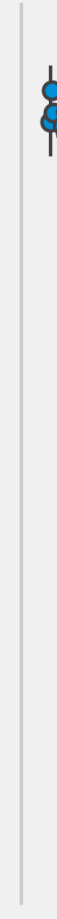

0.00

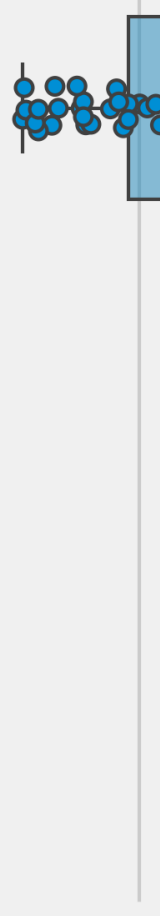

0.25

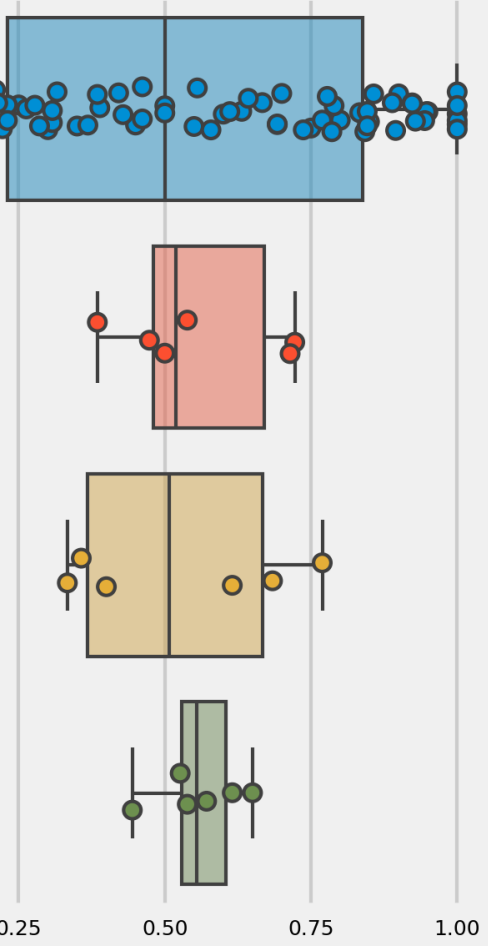

Scaled rank
B.
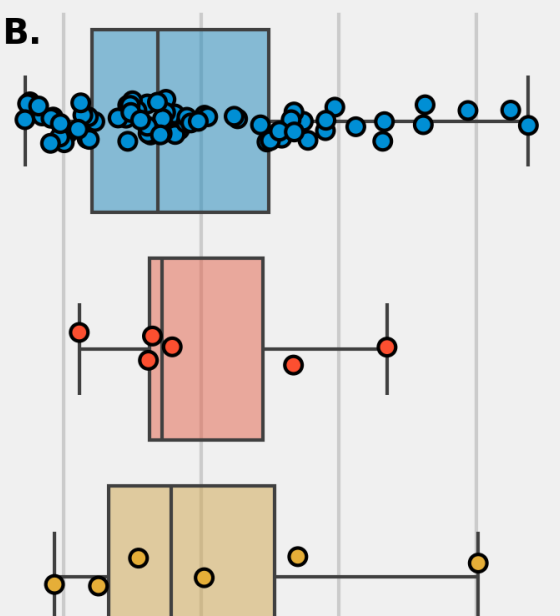

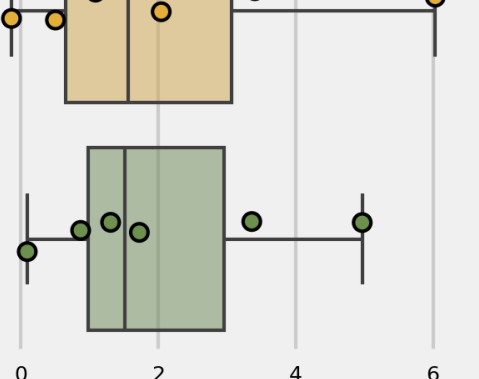

log score

FIG. 7: (A.) The scaled rank across all questions where the truth could be determined for individual trained forecasters and experts, a consensus of trained forecasters, consensus of experts, and consensus of both trained forecasters and experts. (B.) The log score across all questions for individuals and the same three consensus predictions. Individual ranks and scores have a larger variability than any of the three consensus forecasts. The consensus of trained forecasters and experts has the smallest interquartile range for scaled rank and second smallest interquartile range for log score. A consensus forecast is often in the top 50th most accurate forecasts and has consistent performance. 
[1] Angel N Desai and Payal Patel. Stopping the spread of covid-19. Jama, 323(15):1516-1516, 2020.

[2] Renyi Zhang, Yixin Li, Annie L Zhang, Yuan Wang, and Mario J Molina. Identifying airborne transmission as the dominant route for the spread of covid-19. Proceedings of the National Academy of Sciences, 117(26):14857$14863,2020$.

[3] Ian F Miller, Alexander D Becker, Bryan T Grenfell, and C Jessica E Metcalf. Disease and healthcare burden of covid-19 in the united states. Nature Medicine, 26(8):1212-1217, 2020.

[4] Marissa G Baker, Trevor K Peckham, and Noah S Seixas. Estimating the burden of united states workers exposed to infection or disease: a key factor in containing risk of covid-19 infection. PloS one, 15(4):e0232452, 2020.

[5] Mohamed Hassany, Wael Abdel-Razek, Noha Asem, Mohamed AbdAllah, and Hala Zaid. Estimation of covid-19 burden in egypt. The Lancet Infectious Diseases, 20(8):896-897, 2020.

[6] Oecd economic outlook, interim report, march 2021. https://www.oecd-ilibrary.org/economics/oecd-econo mic-outlook/volume-2020/issue-2_34bfd999-en.

[7] Nikolaos G Papadopoulos, Adnan Custovic, Antoine Deschildre, Alexander G Mathioudakis, Wanda Phipatanakul, Gary Wong, Paraskevi Xepapadaki, Ioana Agache, Leonard Bacharier, Matteo Bonini, et al. Impact of covid-19 on pediatric asthma: practice adjustments and disease burden. The Journal of Allergy and Clinical Immunology: In Practice, 8(8):2592-2599, 2020.

[8] Ernesto L Schiffrin, John M Flack, Sadayoshi Ito, Paul Muntner, and R Clinton Webb. Hypertension and covid-19, 2020.

[9] Nicholas G Davies, Petra Klepac, Yang Liu, Kiesha Prem, Mark Jit, and Rosalind M Eggo. Age-dependent effects in the transmission and control of covid-19 epidemics. Nature medicine, 26(8):1205-1211, 2020.

[10] Andrew Atkeson. What will be the economic impact of covid-19 in the us? rough estimates of disease scenarios. Technical report, National Bureau of Economic Research, 2020.

[11] Felipe Ornell, Jaqueline B Schuch, Anne O Sordi, and Felix Henrique Paim Kessler. "pandemic fear" and covid-19: mental health burden and strategies. Brazilian Journal of Psychiatry, 42(3):232-235, 2020.

[12] Cato T Laurencin and Aneesah McClinton. The covid-19 pandemic: a call to action to identify and address racial and ethnic disparities. Journal of racial and ethnic health disparities, 7(3):398-402, 2020.

[13] Amirhossein Hessami, Amir Shamshirian, Keyvan Heydari, Fatemeh Pourali, Reza Alizadeh-Navaei, Mahmood Moosazadeh, Saeed Abrotan, Layla Shojaei, Sogol Sedighi, Danial Shamshirian, et al. Cardiovascular diseases burden in covid-19: Systematic review and meta-analysis. The American journal of emergency medicine, 2020.

[14] Adrian Diaz, Benjamin A Sarac, Anna R Schoenbrunner, Jeffrey E Janis, and Timothy M Pawlik. Elective surgery in the time of covid-19. The American Journal of Surgery, 2020.

[15] Clare Bambra, Ryan Riordan, John Ford, and Fiona Matthews. The covid-19 pandemic and health inequalities. J Epidemiol Community Health, 74(11):964-968, 2020.

[16] An Pan, Li Liu, Chaolong Wang, Huan Guo, Xingjie Hao, Qi Wang, Jiao Huang, Na He, Hongjie Yu, Xihong Lin, et al. Association of public health interventions with the epidemiology of the covid-19 outbreak in wuhan, china. Jama, 323(19):1915-1923, 2020.

[17] Shengjie Lai, Nick W Ruktanonchai, Liangcai Zhou, Olivia Prosper, Wei Luo, Jessica R Floyd, Amy Wesolowski, Mauricio Santillana, Chi Zhang, Xiangjun Du, et al. Effect of non-pharmaceutical interventions to contain covid19 in china. Nature, 585(7825):410-413, 2020.

[18] Nicholas G Davies, Adam J Kucharski, Rosalind M Eggo, Amy Gimma, W John Edmunds, Thibaut Jombart, Kathleen O'Reilly, Akira Endo, Joel Hellewell, Emily S Nightingale, et al. Effects of non-pharmaceutical interventions on covid-19 cases, deaths, and demand for hospital services in the uk: a modelling study. The Lancet Public Health, 5(7):e375-e385, 2020.

[19] Howard Markel, Harvey B Lipman, J Alexander Navarro, Alexandra Sloan, Joseph R Michalsen, Alexandra Minna Stern, and Martin S Cetron. Nonpharmaceutical interventions implemented by us cities during the 1918-1919 influenza pandemic. Jama, 298(6):644-654, 2007.

[20] Corey M Peak, Lauren M Childs, Yonatan H Grad, and Caroline O Buckee. Comparing nonpharmaceutical interventions for containing emerging epidemics. Proceedings of the National Academy of Sciences, 114(15):40234028, 2017. 
[21] Richard J Hatchett, Carter E Mecher, and Marc Lipsitch. Public health interventions and epidemic intensity during the 1918 influenza pandemic. Proceedings of the National Academy of Sciences, 104(18):7582-7587, 2007.

[22] Min W Fong, Huizhi Gao, Jessica Y Wong, Jingyi Xiao, Eunice YC Shiu, Sukhyun Ryu, and Benjamin J Cowling. Nonpharmaceutical measures for pandemic influenza in nonhealthcare settings - social distancing measures. Emerging infectious diseases, 26(5):976, 2020.

[23] Nicola Perra. Non-pharmaceutical interventions during the covid-19 pandemic: A review. Physics Reports, 2021.

[24] John R Mascola and Anthony S Fauci. Novel vaccine technologies for the 21st century. Nature Reviews Immunology, 20(2):87-88, 2020.

[25] Roy Curtiss et al. Bacterial infectious disease control by vaccine development. The Journal of clinical investigation, 110(8):1061-1066, 2002.

[26] Lea Skak Filtenborg Frederiksen, Yibang Zhang, Camilla Foged, and Aneesh Thakur. The long road toward covid19 herd immunity: vaccine platform technologies and mass immunization strategies. Frontiers in immunology, $11,2020$.

[27] Seyed M Moghadas, Thomas N Vilches, Kevin Zhang, Chad R Wells, Affan Shoukat, Burton H Singer, Lauren Ancel Meyers, Kathleen M Neuzil, Joanne M Langley, Meagan C Fitzpatrick, et al. The impact of vaccination on covid-19 outbreaks in the united states. medRxiv, 2020.

[28] Sen Pei, Sasikiran Kandula, and Jeffrey Shaman. Differential effects of intervention timing on covid-19 spread in the united states. Science advances, 6(49):eabd6370, 2020.

[29] Fernando P Polack, Stephen J Thomas, Nicholas Kitchin, Judith Absalon, Alejandra Gurtman, Stephen Lockhart, John L Perez, Gonzalo Pérez Marc, Edson D Moreira, Cristiano Zerbini, et al. Safety and efficacy of the bnt162b2 mrna covid-19 vaccine. New England Journal of Medicine, 383(27):2603-2615, 2020.

[30] Lindsey R Baden, Hana M El Sahly, Brandon Essink, Karen Kotloff, Sharon Frey, Rick Novak, David Diemert, Stephen A Spector, Nadine Rouphael, C Buddy Creech, et al. Efficacy and safety of the mrna-1273 sars-cov-2 vaccine. New England Journal of Medicine, 384(5):403-416, 2021.

[31] Jerald Sadoff, Mathieu Le Gars, Georgi Shukarev, Dirk Heerwegh, Carla Truyers, Anne M de Groot, Jeroen Stoop, Sarah Tete, Wim Van Damme, Isabel Leroux-Roels, et al. Interim results of a phase 1-2a trial of ad26. cov2. s covid-19 vaccine. New England Journal of Medicine, 2021.

[32] Cdc: Covid-19 vaccinations in the united states. https://covid.cdc.gov/covid-data-tracker/\#vaccinations.

[33] Covid-19 vaccine and therapeutics expert predictions. https://github.com/computationalUncertaintyLab/v accinceAndTherapeuticsCrowd.

[34] Natalie E Dean, Ana Pastore y Piontti, Zachary J Madewell, Derek AT Cummings, Matthew DT Hitchings, Keya Joshi, Rebecca Kahn, Alessandro Vespignani, M Elizabeth Halloran, and Ira M Longini Jr. Ensemble forecast modeling for the design of covid-19 vaccine efficacy trials. Vaccine, 38(46):7213-7216, 2020.

[35] Path to herd immunity - covid-19 vaccine projections. https://covid19-projections.com/path-to-herd-imm unity/.

[36] Thomas Charles McAndrew and Nicholas G Reich. An expert judgment model to predict early stages of the covid-19 outbreak in the united states. Medrxiv, 2020.

[37] Steve Lloyd Laura Subramanian Kalipso Chalkidou Adrian La Porta Jiabin Li Eddine Maiza David Reader Julie Rosenberg Jack Scannell Vaughan Thomas Rebecca Weintraub Anthony McDonnell, Robert Van Exan and publisher=Center for Global Development Prashant Yadav journal=CGD Policy Paper, year=2020. Covid-19 vaccine predictions: Using mathematical modelling and expert opinions to estimate timelines and probabilities of success of covid-19 vaccines.

[38] Rapid: Time-sensitive human forest and model forecasts for covid-19 vaccine and treatment trials. https: //www.nsf .gov/awardsearch/showAward?AWD_ID=2030015.

[39] Roger Cooke. Combining expert opinions. In Experts in Uncertainty, pages 171-175. Oxford University Press, 1991.

[40] Nicholas G Reich, Logan C Brooks, Spencer J Fox, Sasikiran Kandula, Craig J McGowan, Evan Moore, Dave Osthus, Evan L Ray, Abhinav Tushar, Teresa K Yamana, et al. A collaborative multiyear, multimodel assessment of seasonal influenza forecasting in the united states. Proceedings of the National Academy of Sciences, 116(8):3146-3154, 2019.

[41] Robert Clemen. Combining forecasts: A review and annotated bibliography. International Journal of Forecasting, 5:559-583, 1989. 
[42] Thomas McAndrew, Nutcha Wattanachit, Graham C Gibson, and Nicholas G Reich. Aggregating predictions from experts: A review of statistical methods, experiments, and applications. Wiley Interdisciplinary Reviews: Computational Statistics, 13(2):e1514, 2021.

[43] Anca M Hanea. Expert Judgement in Risk and Decision Analysis. Springer Nature, 2021.

[44] Robert L Winkler, Yael Grushka-Cockayne, Kenneth C Lichtendahl Jr, and Victor Richmond R Jose. Probability forecasts and their combination: A research perspective. Decision Analysis, 16(4):239-260, 2019.

[45] Barbara Mellers, Lyle Ungar, Jonathan Baron, Jaime Ramos, Burcu Gurcay, Katrina Fincher, Sydney E Scott, Don Moore, Pavel Atanasov, Samuel A Swift, et al. Psychological strategies for winning a geopolitical forecasting tournament. Psychological science, 25(5):1106-1115, 2014.

[46] David C Farrow, Logan C Brooks, Sangwon Hyun, Ryan J Tibshirani, Donald S Burke, and Roni Rosenfeld. A human judgment approach to epidemiological forecasting. PLoS computational biology, 13(3):e1005248, 2017.

[47] Nicholas B DeFelice, Eliza Little, Scott R Campbell, and Jeffrey Shaman. Ensemble forecast of human west nile virus cases and mosquito infection rates. Nature Communications, 8(1):1-6, 2017.

[48] Madeleine C Thomson, FJ Doblas-Reyes, Simon J Mason, Renate Hagedorn, Stephen J Connor, Thandie Phindela, AP Morse, and TN Palmer. Malaria early warnings based on seasonal climate forecasts from multi-model ensembles. Nature, 439(7076):576-579, 2006.

[49] Katherine F Smith, Andrew P Dobson, F Ellis McKenzie, Leslie A Real, David L Smith, and Mark L Wilson. Ecological theory to enhance infectious disease control and public health policy. Frontiers in Ecology and the Environment, 3(1):29-37, 2005.

[50] Ralph J DiClemente, Laura Francisca Salazar, and Richard A Crosby. Health behavior theory for public health: Principles, foundations, and applications. Jones \& Bartlett Publishers, 2013.

[51] Ronan F Arthur, Emily S Gurley, Henrik Salje, Laura SP Bloomfield, and James H Jones. Contact structure, mobility, environmental impact and behaviour: the importance of social forces to infectious disease dynamics and disease ecology. Philosophical Transactions of the Royal Society B: Biological Sciences, 372(1719):20160454, 2017.

[52] Carl Latkin, Lauren A Dayton, Grace Yi, Arianna Konstantopoulos, Ju Park, Catherine Maulsby, and Xiangrong Kong. Covid-19 vaccine intentions in the united states, a social-ecological framework. Vaccine, 2021.

[53] Azeem Majeed and Mariam Molokhia. Vaccinating the uk against covid-19, 2020.

[54] Abu Raihan Bhuiyan Albarune and Md Mamun Habib. A study of forecasting practices in supply chain management. International Journal of Supply Chain Management, 4(2):55-61, 2015.

[55] Aris A Syntetos, Zied Babai, John E Boylan, Stephan Kolassa, and Konstantinos Nikolopoulos. Supply chain forecasting: Theory, practice, their gap and the future. European Journal of Operational Research, 252(1):1-26, 2016.

[56] Michael J Rogers, Anshuman Gupta, and Costas D Maranas. Real options based analysis of optimal pharmaceutical research and development portfolios. Industrial $\mathcal{G}$ engineering chemistry research, 41(25):6607-6620, 2002.

[57] Xiaoxia Huang, Tianyi Zhao, and Shamsiya Kudratova. Uncertain mean-variance and mean-semivariance models for optimal project selection and scheduling. Knowledge-Based Systems, 93:1-11, 2016.

[58] Farhad Hassanzadeh, Mohammad Modarres, Hamid R Nemati, and Kwasi Amoako-Gyampah. A robust r\&d project portfolio optimization model for pharmaceutical contract research organizations. International Journal of Production Economics, 158:18-27, 2014.

[59] Majid Shakhsi-Niaei, S Ali Torabi, and Seyed Hossein Iranmanesh. A comprehensive framework for project selection problem under uncertainty and real-world constraints. Computers \& Industrial Engineering, 61(1):226$237,2011$.

[60] Metaculus. https://www.metaculus.com/questions/, 2020. [Online; accessed May 13, 2021].

[61] Amos Tversky and Daniel Kahneman. Judgment under uncertainty: Heuristics and biases. science, 185(4157):1124-1131, 1974.

[62] Barbara Mellers, Eric Stone, Terry Murray, Angela Minster, Nick Rohrbaugh, Michael Bishop, Eva Chen, Joshua Baker, Yuan Hou, Michael Horowitz, et al. Identifying and cultivating superforecasters as a method of improving probabilistic predictions. Perspectives on Psychological Science, 10(3):267-281, 2015.

[63] Philip E Tetlock and Dan Gardner. Superforecasting: The art and science of prediction. Random House, 2016.

[64] Barbara Mellers, Eric Stone, Pavel Atanasov, Nick Rohrbaugh, S Emlen Metz, Lyle Ungar, Michael M Bishop, 
Michael Horowitz, Ed Merkle, and Philip Tetlock. The psychology of intelligence analysis: Drivers of prediction accuracy in world politics. Journal of experimental psychology: applied, 21(1):1, 2015.

[65] Christian Genest and Kevin J McConway. Allocating the weights in the linear opinion pool. Journal of Forecasting, 9(1):53-73, 1990.

[66] Irving John Good. Rational decisions. In Breakthroughs in statistics, pages 365-377. Springer, 1992.

[67] Mark S Roulston and Leonard A Smith. Combining dynamical and statistical ensembles. Tellus A: Dynamic Meteorology and Oceanography, 55(1):16-30, 2003.

[68] Tilmann Gneiting and Adrian E Raftery. Strictly proper scoring rules, prediction, and estimation. Journal of the American statistical Association, 102(477):359-378, 2007.

[69] Tilmann Gneiting, Fadoua Balabdaoui, and Adrian E Raftery. Probabilistic forecasts, calibration and sharpness. Journal of the Royal Statistical Society: Series B (Statistical Methodology), 69(2):243-268, 2007.

[70] Merryn Voysey, Sue Ann Costa Clemens, Shabir A Madhi, Lily Y Weckx, Pedro M Folegatti, Parvinder K Aley, Brian Angus, Vicky L Baillie, Shaun L Barnabas, Qasim E Bhorat, et al. Safety and efficacy of the chadox1 ncov-19 vaccine (azd1222) against sars-cov-2: an interim analysis of four randomised controlled trials in brazil, south africa, and the uk. The Lancet, 397(10269):99-111, 2021.

[71] The RECOVERY Collaborative Group. Dexamethasone in hospitalized patients with covid-19. New England Journal of Medicine, 384(8):693-704, 2020.

[72] Chi Heem Wong, Kien Wei Siah, and Andrew Lo. Estimation of clinical trial success rates and related parameters. Biostatistics, 20(2):273-286, 2018.

[73] Egon Brunswik. Perception and the representative design of psychological experiments. Univ of California Press, 1956. 
medRxiv preprint doi: https://doi.org/10.1101/2021.06.03.21258240; this version posted June 4, 2021. The copyright holder for this preprint (which was not certified by peer review) is the author/funder, who has granted medRxiv a license to display the preprint in perpetuity.

It is made available under a CC-BY-NC-ND 4.0 International license.

VII. SUPPLEMENTAL MATERIALS

VIII. QUESTIONS ASKED FOR ALL FOUR SURVEYS 
Dear <Expert>,

We read your work on <insert work $>$ with great interest, and invite you to join a collaborative group of select experts.

We are building expert consensus predictions about the development of SARSCoV-2 vaccines and COVID-19 therapeutics each month with a small group of researchers involved in the study of novel therapeutics/vaccines. Experts are surveyed about their predictions about the future development of SARS-CoV-2 vaccines and COVID-19 therapeutics and the results are bei aggregated into a consensus.

We feel your skill set defines you as an expert in this field. Would you participate, alongside other experts, in our survey project to forecast the development of SARS-CoV-2 vaccines and COVID-19 therapeutics?

Your anonymized predictions will contribute to an expert consensus made available to the public, and sent to the CDC to provide support for public health decision making. Our main goal is to provide public health officials probabilistic predictions from experts on the research and development of vaccines and therapeutics. We have made the results from our first survey available here

Our past work, in collaboration with Thomas McAndrew at the University of Massachusetts at Amherst, focused on forecasting the early trajectory of COVID-19 in the United States. Forecasts and predictions generated by 41 experts in the modeling of infectious disease were featured in outlets such as Science FiveThirtyEight and The Economist, and were also sent to the CDC to support the US COVID-19 response.

If you are available to participate in at least one survey, please respond to this email, preferably by $<M M D D>$. We expect to administer the next monthly survey on $<$ MMDD $>$. We would be thrilled to welcome you-if only just for one round. An expert consensus can produce forecasts on a diverse range of vaccine and therapeutic solutions that computational and statistical methods cannot, and we feel your expertise will make impactful and meaningful contributions.

Please reach out to us with any questions.

\section{Sincerely,}

FIG. S1: A template email used to solicit forecasting participation from subject matter experts in molecular and cellular biology, microbiology, virology, biochemistry, and infectious disease who have had several years of experience studying vaccine, antiviral, or biological related to infectious agents. 


\section{Question}

\section{Survey 1 (June, 2020)}

What will be the efficacy of the Oxford/AstraZeneca ChAdOx1 nCoV-19 vaccine candidate according to the results of Phase II/III testing?

When will the first SARS-CoV-2 vaccine to be approved in the US or EU be administered to >100K people?

How many SARS-CoV-2 vaccine candidates will be in human trials as of 1 August 2020?

When will a SARS-CoV-2 vaccine candidate be approved for use in the United States or European Union?

When will a SARS-CoV-2 vaccine candidate demonstrate $\geq 70 \%$ efficacy?

When will a COVID-19 therapeutic or therapeutics cocktail show a statistically significant survival benefit for the treatment group in a $\mathrm{n}>200 \mathrm{RCT}$ ?

\section{Survey 2 (July, 2020)}

When will a SARS-CoV-2 antiviral show a statistically significant survival benefit for the treatment group in an $\mathrm{n}_{\llcorner} 200$ RCT? What will be the efficacy of the first US- or EU- approved SARS-CoV-2 vaccine based on a non-replicating viral vector platform?

When will a SARS-CoV-2 monoclonal antibody or antibody cocktail show a statistically significant survival benefit for the treatment group in an $\mathrm{n}>200$ RCT?

What will be the efficacy of the first US- or EU- approved SARS-CoV-2 vaccine based on a protein subunit platform? What will be the efficacy of the first US- or EU- approved SARS-CoV-2 vaccine based on an inactivated virus platform? What will be the efficacy of the first US- or EU- approved SARS-CoV-2 vaccine based on a DNA or RNA platform? When will a SARS-CoV-2 vaccine candidate be approved for use in the United States or European Union?

\section{Survey 3 (August, 2020)}

When will a SARS-CoV-2 vaccine candidate be approved for use in the US or EU through a normal approval process? When will a SARS-CoV-2 vaccine candidate be approved for use in the US or EU through an emergency approval process?

What will be the efficacy of the first US- or EU- approved SARS-CoV-2 vaccine candidate approved through a normal approval process?

What will be the efficacy of the first US- or EU- approved SARS-CoV-2 vaccine candidate approved through an emergency approval process?

How many weeks after approval will the first 100 million doses of the first US- or EU- approved SARS-CoV-2 vaccine candidate based on a DNA or RNA platform be manufactured?

How soon after approval will the first 100 million doses of the first US- or EU- approved SARS-CoV-2 vaccine candidate based on a non-replicating viral vector platform be manufactured?

When will an orally administered SARS-CoV-2 antiviral show a statistically significant survival benefit for the treatment group in an $\mathrm{n}>200 \mathrm{RCT}$ ?

What will be the SARS-CoV-2 infectivity of children relative to adults when schools are open?

Survey 4 (September, 2020)

When will a SARS-CoV-2 vaccine candidate be approved for use in the US through a normal approval process? When will a SARS-CoV-2 vaccine candidate be approved for use in the US through an emergency approval process? What will be the efficacy ratio of the first SARS-CoV-2 vaccine candidate approved on an emergency basis (numerator) compared to the first approved through a normal process (denominator)?

What is the probability of at least ten serious adverse events (SAEs) being attributed within one year to the first SARS-CoV-2 vaccine approved in the US through a normal approval process?

What is the probability of at least ten serious adverse events (SAEs) being attributed within one year to the first SARS-CoV-2 vaccine approved in the US through an emergency approval process?

TABLE S1: List of all questions stratified by survey. 